\title{
Electronic Transport through Magnetic Molecules with Soft Vibrating Modes
}

\author{
P. S. Cornaglia, ${ }^{1,2}$ Gonzalo Usaj, ${ }^{1}$ and C. A. Balseiro ${ }^{1}$ \\ ${ }^{I}$ Instituto Balseiro and Centro Atómico Bariloche, Comisión Nacional de Energía Atómica, 8400 San Carlos de Bariloche, Argentina. \\ ${ }^{2}$ Centre de Physique Théorique, École Polytechnique, CNRS, 91128 Palaiseau Cedex, France.
}

(Dated: September 26, 2007)

\begin{abstract}
The low-temperature transport properties of a molecule are studied in the field-effect transitor geometry. The molecule has an internal mechanical mode that modulates its electronic levels and renormalizes both the interactions and the coupling to the electrodes. For a soft mechanical mode the spin fluctuations in the molecule are dominated by the bare couplings while the valence changes are determined by the dressed energies. In this case, the transport properties present an anomalous behavior and the Kondo temperature has a weak gate voltage dependence. These observations are in agreement with recent experimental data.
\end{abstract}

PACS numbers: 72.15.Qm, 73.22.-f

The recent development of molecular transistors (MT) has created new scenarios for the study of correlation effects in nanoscopic systems. These devices have attracted a lot of interest due to their potential application in nanoelectronics and their rich variety of behavior. Molecular transistors consist of a small molecule connecting two electrodes and, in most cases, a gate electrode is used to control the molecule's charge electrostatically. The transport properties of MT show signatures of strong electronic correlations as Coulomb blockade [1] and the Kondo effect [2, 3, 4, 5] similar to those observed in quantum dot devices (QD) [6, 7]. A remarkable difference between QD and MT is the coupling, on the latter, of the electronic degrees of freedom with a discrete set of mechanical modes [8, 9, 10].

For the simplest case of a linear modulation of the molecule's electronic levels by a single vibration mode, several effects are predicted to occur. The Franck-Condon renormalization of the molecule-electrodes coupling is expected to produce a suppression in the sequential and cotunneling transport through the molecule [11, 12, 13]. The reduction of the effective Coulomb repulsion in the molecule may lead to an effective e-e attraction [14, 15, 16] and a strong sensitivity to gate voltage.

In the repulsive e-e interaction regime the spin-Kondo effect dominates the low-temperature physics. The Kondo effect generates in these devices an increase of the conductance with decreasing temperature and a zero-bias peak in the differential conductance. These observations are a direct consequence of the formation of the Abrikosov-Suhl or Kondo resonance below the Kondo temperature $T_{K}$ [17].

In some MTs based on organometallic molecules, an anomalous gate voltage dependence of the transport properties has been reported [1, 2, 5]. In the transition metal complexes studied by Yu et al. [5], $T_{K}$ depends weakly on the applied gate voltage and shows a rapid increase only close to the charge degeneracy points. Moreover, the edges of the Coulomb blockade diamonds are not well defined in the Kondo charge state. Such a behavior is inconsistent with the usual theory based on the Anderson model.

In this Rapid Communication we present a study of the Anderson-Holstein model showing results obtained with the numerical renormalization group (NRG) [18, 19]. We find that, as the frequency of the vibrating mode decreases, an anomalous gate dependence of $T_{K}$ and of the transport properties emerges. This effect arises because the soft vibrating modes in the MT drive the system into a new regime where the characteristic energy scales for spin and charge fluctuations are not related as in the conventional theory of the Kondo effect.

The model Hamiltonian is $H=H_{M}+H_{E}+H_{M E}$ where the first two terms describe the isolated molecule and the electrodes, respectively, and the last term describes their coupling. We have

$$
\begin{aligned}
H_{M} & =\varepsilon_{d} n_{d}+U n_{d \uparrow} n_{d \downarrow}-\lambda\left(n_{d}-1\right)\left(a+a^{\dagger}\right)+\omega_{0} a^{\dagger} a \\
H_{E} & =\sum_{k, \sigma, \alpha} \varepsilon_{\alpha k} c_{\alpha k \sigma}^{\dagger} c_{\alpha k \sigma}, \\
H_{M E} & =\sum_{k, \sigma, \alpha} V_{\alpha k}\left(d_{\sigma}^{\dagger} c_{\alpha k \sigma}+c_{\alpha k \sigma}^{\dagger} d_{\sigma}\right) .
\end{aligned}
$$

Here $n_{d}=n_{d \uparrow}+n_{d \downarrow}, n_{d \sigma}=d_{\sigma}^{\dagger} d_{\sigma}, d_{\sigma}^{\dagger}$ creates an electron at the molecular orbital with energy $\varepsilon_{d}, U$ is the intramolecular Coulomb repulsion and $c_{\alpha k \sigma}^{\dagger}$ creates an electron in the mode $k$ of electrode $\alpha=L, R$. The operator $a^{\dagger}$ creates an excitation of the vibronic mode with energy $\omega_{0}$. The Fermi energy $E_{F}$ is set to zero, $\hbar=1$, and all energies are in units of half the electrodes' bandwidth. For the sake of simplicity, from here on we consider a symmetric molecule with identical $L$ and $R$ electrodes $\left(\varepsilon_{\alpha k} \equiv \varepsilon_{k}\right)$ and take $V_{L k}=V_{R k} \equiv V_{h y b}$.

The energy spectrum of the isolated molecule $\left(V_{h y b}=0\right)$ can be readily obtained. The energies for the $n_{d}=0,1$ and 2 charge states, with $m$ vibron excitations, are $E_{0, m}=-\lambda^{2} /$ $\omega_{0}+m \omega_{0}, E_{1, m}=\varepsilon_{d}+m \omega_{0}$, and $E_{2, m}=-\lambda^{2} / \omega_{0}+2 \varepsilon_{d}+$ $U+m \omega_{0}$, respectively. The corresponding states are indicated as $|0, m\rangle,|\sigma, m\rangle$, and $|2, m\rangle$ where $\sigma$ is the spin index in the $n_{d}=1$ charge sector-note that the vibronic states for different charge states correspond to different equilibrium positions of the coordinate associated with the vibronic motion. It is convenient to define the effective single-electron energy and $e$-e repulsion as $\widetilde{\varepsilon}_{d}=E_{1,0}-E_{0,0}=\varepsilon_{d}+\lambda^{2} / \omega_{0}$ and $\widetilde{U}=E_{2,0}+E_{0,0}-2 E_{1,0}=U-2 \lambda^{2} / \omega_{0}$, respectively. The charge degeneracy points of the isolated molecule are given 

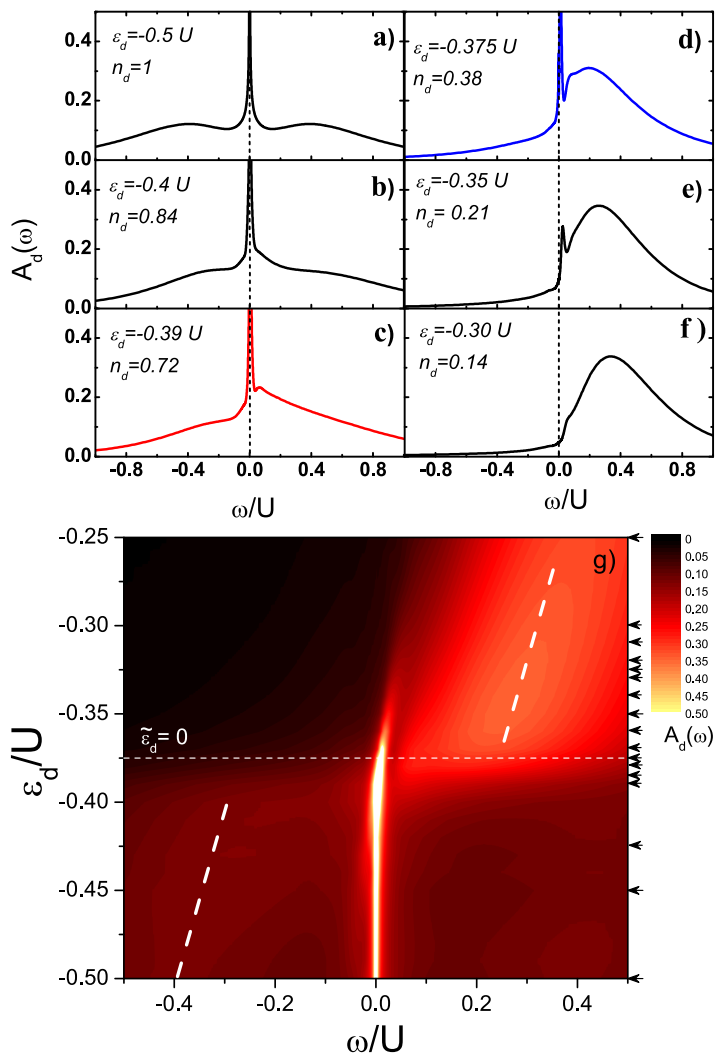

FIG. 1: (color online) Spectral density $A_{d}(\omega)$ (in units of $1 / \pi \Gamma$ ). Panel a) to f) $A_{d}(\omega)$ vs $\omega$ for $U=0.2, V_{h y b}=0.1 / \sqrt{2}, g=$ $6.25, \lambda=0.03$ and different values of $\varepsilon_{d}$. g) Color map of $A_{d}(\omega)$ constructed by linear extrapolation of the NRG results obtained for different values of $\varepsilon_{d}$ (indicated by arrows). Thick dashed lines are a guide to the eye of the form $\varepsilon_{d}=\omega+a$ and the horizontal line indicates the charge degeneracy point for the isolated molecule $\left(\widetilde{\varepsilon}_{d}=\right.$ $0)$. Values of $A_{d}(\omega)$ larger than 0.5 are white in the color scale.

by $\widetilde{\varepsilon}_{d}=0$ and $\widetilde{\varepsilon}_{d}+\widetilde{U}=0$. When the molecule is coupled to the electrodes, the charge fluctuations are controlled by the Franck-Condon (FC) factors $\gamma_{n, m}=\left|\left\langle\sigma, n\left|d_{\sigma}^{\dagger}\right| 0, m\right\rangle\right|^{2}$. We define $\gamma_{m} \equiv \gamma_{0, m}=e^{-g} g^{m} / m$ !, where $g=\left(\lambda / \omega_{0}\right)^{2}$ is a dimensionless parameter. For $g \gg 1$, transitions between low-lying states are exponentially suppressed and the system is in the FC blockade regime [11, 12].

We now present numerical results for the molecular spectral density $A_{d}(\omega)$ which determines the transport properties of the MT. $A_{d}(\omega)$ is given by $-(1 / \pi) \operatorname{Im} G_{d d}(\omega)$, where $G_{d d}(\omega)$ is the electronic Green's function of the molecule in the presence of the electrodes. The zero-temperature NRG results for $A_{d}(\omega)$ are shown in Fig. 1 for different values of the parameters in a regime of strong FC effect $(g=6.25)$. In the electron-hole symmetric case $\left[\varepsilon_{d}=-U / 2\right.$, Fig. [1)], $A_{d}(\omega)$ shows broad structures at the bare energies $\varepsilon_{d}$ and $\varepsilon_{d}+U$ and a well defined Kondo peak at the Fermi level. Although not well resolved in the figure, peaks are also obtained at $\widetilde{\varepsilon}_{d}$ and $\widetilde{\varepsilon}_{d}+\widetilde{U}$-these are the first FC sidebands. The evolution of the spectral density $A_{d}(\omega)$ as the molecular energy is shifted up- wards presents interesting features. First, the broad peaks and the first FC peaks-which are better resolved for small $\left|\widetilde{\varepsilon}_{d}\right|$ [see Fig. 15)]- shift with the molecular energy, while the Kondo temperature, as given by the width of the Kondo peak, is not very sensitive to it. As $\widetilde{\varepsilon}_{d}$ crosses $E_{F}$, the behavior of $A_{d}(\omega)$ indicates a rapid change in the occupation of the molecular orbital $\left\langle n_{d}\right\rangle$. There is no crossing of a wide resonance through the Fermi level, as it occurs in the $g=0$ case. Here instead, there is a rapid transfer of spectral weight from $\omega \sim \varepsilon_{d}$ to $\omega \sim \varepsilon_{d}+U$, in agreement with the atomic limit results [19]. The change in the structure of $A_{d}(\omega)$ occurs as the Kondo peak evolves into a FC peak at small $\omega$. This is better seen in the color map of the spectral density shown in Fig. 1 $1 \mathrm{~g}$ ). This anomalous behavior has important consequences on the thermodynamic and transport properties of the system.

The occupation of the molecular orbital versus the bare energy $\varepsilon_{d}$ is shown in Fig. 2a) for different values of $g$. As $g$ increases, the width of the region where the magnetic configuration is stable $\left(\left\langle n_{d}\right\rangle \simeq 1\right)$ and the crossover widths between the different charge states decrease. The former is due to the reduction of the effective $e$-e repulsion $\widetilde{U}$ while the latter, being exponential with $g$, is a manifestation of FC effects [11, 12]. Similar FC effects are obtained in the spinless case [11]. However, for the Anderson-Holstein model presented here, the interplay between $e-e$ and $e-v$ interactions leads to a crossover between different charge states that is wider than in the spinless case. Also, for the spinfull model with large $g$ the charge degeneracy points (with $\left\langle n_{d}\right\rangle=1 / 2$ and $3 / 2$ ) are not given exactly by $\widetilde{\varepsilon}_{d}=0$ and $\widetilde{\varepsilon}_{d}+\widetilde{U}=0$ as self-energy corrections, due to the hybridization, shift the dressed molecular levels.

The magnetic susceptibility $\chi(T)$ of the molecule is shown in Fig. (2b) for different values of $\epsilon_{d}$. In the Kondo regime, the low-temperature susceptibility curves collapse into a single curve when the temperature is properly scaled. To calculate the Kondo temperature $T_{K}$ and its dependence on the molecular energy $\varepsilon_{d}$ we use Wilson's criterion [20]: $T_{K} \chi\left(T_{K}\right) / \mu_{B}{ }^{2}=0.025$. The results for $T_{K}$ are shown in Fig. 2r). As $\omega_{0}$ decreases (we keep $\lambda$ fixed and therefore $g$ increases), $T_{K}$ vs. $\widetilde{\varepsilon}_{d}$ deviates from the usual behavior: it shows a weak dependence on $\widetilde{\varepsilon}_{d}$ at the center of the Coulomb blockade region and a fast increase close to the charge degeneracy points.

In terms of the Kondo coupling $J$ the Kondo temperature is given by $T_{K}=D e^{-1 / \rho_{0} J}$ where $D$ is a high energy cutoff and $\rho_{0}$ is the bare electronic density of states of the electrodes at the Fermi level. While the value of $J$ obtained using second order perturbation theory in $V_{h y b}$ underestimates the ratio $T_{K} / T_{K}^{0}$ for small values of $\omega_{0}$, it is instructive to use this approach for a qualitative interpretation of the numerical results. We have [21, 22]

$$
J=\sum_{m=0}\left(\frac{2 V_{h y b}^{2} \gamma_{m}}{-\widetilde{\varepsilon}_{d}+\omega_{0} m}+\frac{2 V_{h y b}^{2} \gamma_{m}}{\widetilde{\varepsilon}_{d}+\widetilde{U}+\omega_{0} m}\right) .
$$

For large $g$, the FC factor $\gamma_{m}$ is peaked at $m \sim m^{\star}=g$ with a width of the order of $\sqrt{m^{\star}}$. As in the summation of 


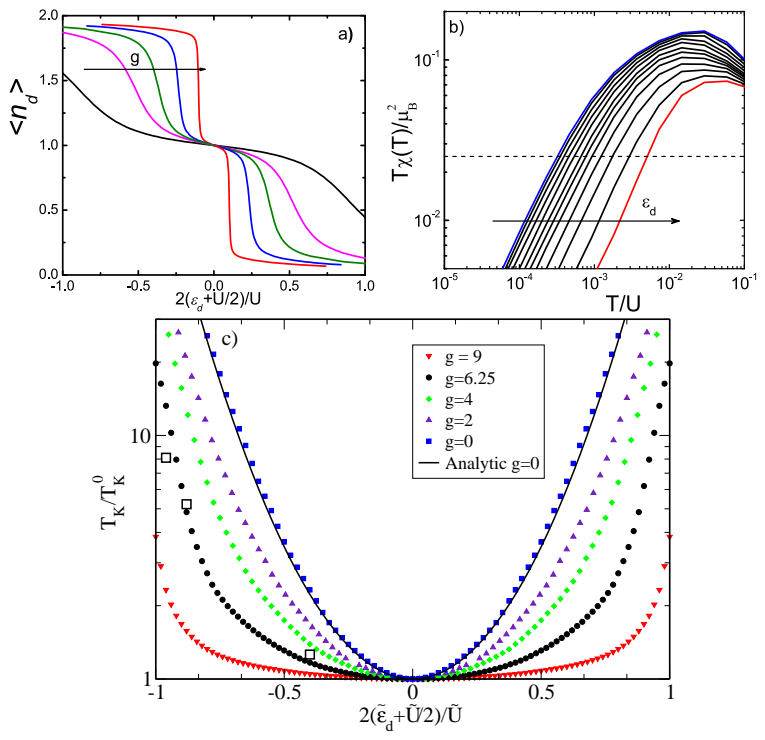

FIG. 2: (color online) a) Occupation of the molecular orbital $\left\langle n_{d}\right\rangle$ vs $\varepsilon_{d}$ for $g=0,2,4,6.25$ and 9 . b) Susceptibility $T \chi(T) / \mu_{B}{ }^{2}$ vs $T$ in a $\log -\log$ plot for $\varepsilon_{d} / U$ increasing from -0.5 to -0.38 , and $g=6.25$. Dashed line indicates Wilson's value. Other parameters as in Fig. 1 c) Kondo temperature $T_{K} / T_{K}^{0}$ vs $\varepsilon_{d}$ for different values of $g$ as indicated in the inset. Open squares are the values of $T_{K}$ obtained by fitting the temperature dependence of the conductance for $g=6.25$ (see text).

Eq. (4) the denominators are slowly varying around $m^{*}$, the Kondo coupling can be approximated as $J \simeq 2 V_{h y b}^{2}\left[1 /\left(-\widetilde{\varepsilon}_{d}+\right.\right.$ $\left.\left.m^{\star} \omega_{0}\right)+1 /\left(\widetilde{U}+\widetilde{\varepsilon}_{d}+m^{\star} \omega_{0}\right)\right]$. This shows that the relevant virtual charge fluctuations have a characteristic energy given by the bare molecular parameters $\varepsilon_{d}=\widetilde{\varepsilon}_{d}-\omega_{0} m^{*}$ and $\varepsilon_{d}+U=\widetilde{\varepsilon}_{d}+\widetilde{U}+\omega_{0} m^{*}$ corresponding to the broad peaks in Fig. 11). However, as $\widetilde{\varepsilon}_{d} \rightarrow 0(-\widetilde{U})$ the $m=0$ term in Eq. (4) diverges with an exponentially small prefactor $e^{-g}$, indicating that the perturbation theory breaks down for $\widetilde{\varepsilon}_{d}$ exponentially close to the charge degeneracy points of the isolated molecule. In other words, for small $\omega_{0}$ and large $g$, the virtual charge fluctuations leading to the Kondo coupling are antiadiabatic while the charge instabilities are controlled by the dressed energies $\widetilde{\varepsilon}_{d}$ and $\widetilde{\varepsilon}_{d}+\widetilde{U}$.

We stress that, even far from the degeneracy points, the perturbative approach of Eq. (4) gives a quantitative estimate of $T_{K} / T_{K}^{0}$ only for large values of $\omega_{0} \gtrsim U / 2$. As $\omega_{0}$ decreases the states $|\sigma, m\rangle$ with $m>0$ also participate in the ground state and should be taken into account [23]. We are not aware of any reliable method to properly handle such a case analytically.

The linear conductance $G$ of the molecular junction at zero bias is given by the spectral density $A_{d}(\omega)$ presented above. For symmetric electrodes [24, 25]

$$
G=\left.\frac{d I}{d V}\right|_{V=0}=\frac{e^{2}}{\hbar} \Gamma \int_{-\infty}^{\infty} d \omega\left(-\frac{\partial f(\omega)}{\partial \omega}\right) A_{d}(\omega)
$$

where $f(\omega)$ is the Fermi distribution and $\Gamma=2 \pi \rho_{0} V_{h y b}^{2}$. The

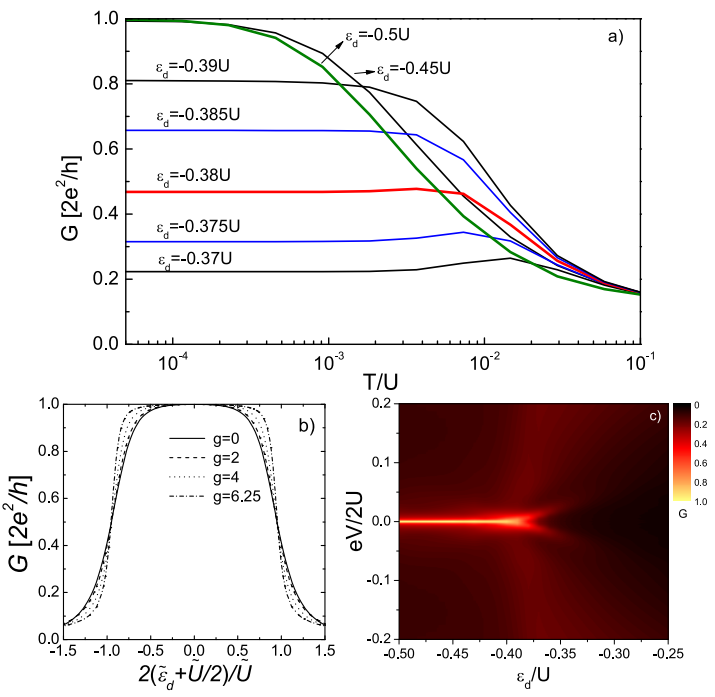

FIG. 3: (color online) a) Temperature dependence of the conductance for different values of $\varepsilon_{d} / U$, and $g=6.25$. The charge degeneracy point is at $\varepsilon_{d} / U \simeq-0.38 U$. b) Zero-temperature conductance vs $\varepsilon_{d}$. Parameters as in Fig. 1 and different values of $g$ as shown in the inset. c) Differential conductance $G(V)$ (in units of $2 e^{2} / h$ ) as a function of the bias and gate voltages (cf. Eq. (7)).

temperature dependence of the conductance for $g=6.25$ and different values of $\varepsilon_{d}$ is presented in Fig. 3a a). In the electron-hole symmetric case $\left(\varepsilon_{d}=-U / 2\right)$ the characteristic Kondo behavior is obtained. As $\varepsilon_{d}$ increases, the low temperature conductance decreases in quantitative agreement with the Fermi liquid zero-temperature results (see below). For $\widetilde{\varepsilon}_{d}>0$ the conductance shows a maximum at a temperature $T^{*}$ consistent with the energy of the first FC side-band. Within the Kondo regime our results for the temperature dependent conductance are well fitted by the phenomenological expression $G(T)=G_{0}^{*}\left[1+\left(2^{1 / s}-1\right)\left(T / T_{K}\right)^{2}\right]^{-s}$ with $s$ between 0.22 and $0.25[6]$. The value of $T_{K}$ obtained with this fitting procedure is consistent with Wilson's criterion as shown in Fig. 2r). It is important to point out that while for $g=0$ the phenomenological expression correctly describes the universal scaling of the conductance even deep into the intermediate valence regime $\left(\left\langle n_{d}\right\rangle \sim 0.5\right)$, it fails to do so for $g \gg 1$ already for $\left\langle n_{d}\right\rangle \simeq 0.75\left(\varepsilon_{d} \simeq-0.39 U\right.$ for the parameters of Fig. 33).

The zero-temperature conductance, shown in Fig. 3b) as a function of $\widetilde{\varepsilon}_{d}$ for different values of $g$, was calculated using [28]

$$
G=\frac{e^{2}}{\hbar} \Gamma A_{d}(0)=\frac{2 e^{2}}{h} \sin ^{2}\left(\frac{\pi\left\langle n_{d}\right\rangle}{2}\right) .
$$

Note that while at high temperatures $\left(T>T_{K}\right)$ the conductance is suppressed by the Coulomb interaction and FranckCondon blockade [13], at low temperatures the Kondo effect sets in and the conductance is high.

A salient feature in the differential conductance of systems with strong $e-v$ coupling is the anomalous behavior of the 
edges of the Coulomb blockade diamonds. In some MT [2, 5] the diamond edges associated with the Kondo charge state $\left(\left\langle n_{d}\right\rangle \simeq 1\right)$ are much weaker, or even absent, than the ones corresponding to the non-Kondo charge state. In the lowest order on the bias voltage, the differential conductance is

$$
G(V) \simeq \frac{e^{2}}{h} \pi \Gamma\left[A_{d}(e V / 2)+A_{d}(-e V / 2)\right]
$$

Although the results obtained with this equation [see Fig. 36)] should only be taken as a rough estimate, they show that the qualitative behavior of the differential conductance is very different from that of the Anderson model. The main reason is that, as discussed above, the valence change is not due to a broad resonant level that crosses $E_{F}$. The spectral weight is rather transferred from below to above $E_{F}$ and only the narrow peak of the first FC band, that has an exponentially reduced weight ( $\propto e^{-g}$ ), crosses $E_{F}$.

In summary, we have studied a model MT where the electronic levels of the molecule are modulated by a well defined internal vibronic mode. We have shown that the coupling to a soft phonon mode $\left(\omega_{0} \ll U\right)$ in the FC regime $(g \gg 1)$ changes qualitatively the behavior of the MT physical properties. The electronic interactions in the molecule and the coupling to the electrodes are strongly renormalized. While spin fluctuations in the molecule are associated to virtual processes dominated by the bare electronic energies, the valence changes occur when the dressed energies cross the Fermi level. This leads to an anomalous gate voltage dependence of the spectral density and $T_{K}$. The theory accounts for the observed weak dependence of $T_{K}$ on gate voltage and suggests the possible origin of the anomalous behavior of the Coulomb blockade diamond edges [5]. We found that the universality characteristic of the Kondo phenomena, is lost in this case much before the valence change as compared with the usual Anderson model. Finally, we showed that the FC suppression of the low-bias conductance expected for the high temperature regime [12, 13] is not present at low temperatures due to the Kondo enhancement of the conductance.

This work was supported by ANPCyT Grants No 13829 and 13476 and CONICET PIP 5254. GU and PSC are members of CONICET (Argentina).

[1] J. Park, A. N. Pasupathy, J. I. Goldsmith, C. Chang, Y. Yaish, J. R. Petta, M. Rinkoski, J. P. Sethna, H. D. Abruña, P. L.
McEuen, et al., Nature 417, 722 (2002).

[2] W. Liang, M. P. Shores, M. Bockrath, J. R. Long, and H. Park, Nature 417, 725 (2002).

[3] A. N. Pasupathy, R. C. Bialczak, J. Martinek, J. E. Grose, L. A. K. Donev, P. L. McEuen, and D. C. Ralph, Science 306, 86 (2004).

[4] L. H. Yu and D. Natelson, Nano Letters 4(1), 79 (2004).

[5] L. H. Yu, Z. K. Keane, J. W. Ciszek, L. Cheng, J. M. Tour, T. Baruah, M. R. Pederson, and D. Natelson, Phys. Rev. Lett. 95, 256803 (2005).

[6] D. Goldhaber-Gordon, H. Shtrikman, D. Mahalu, D. AbuschMagder, U. Meirav, and M. A. Kastner, Nature 391, 156 (1998).

[7] L. P. Kouwenhoven and L. I. Glazman, Physics World January, 33 (2001).

[8] H. Park, J. Park, A. K. L. Lim, E. H. Anderson, A. P. Alivisatos, and P. L. McEuen, Nature 407, 57 (2000).

[9] N. B. Zhitenev, H. Meng, and Z. Bao, Phys. Rev. Lett. 88, 226801 (2002).

[10] X. H. Qiu, G. V. Nazin, and W. Ho, Phys. Rev. Lett. 92, 206102 (2004).

[11] K. Flensberg, Phys. Rev. B 68, 205323 (2003).

[12] J. Koch and F. von Oppen, Phys. Rev. Lett. 94, 206804 (2005).

[13] J. Koch, M. E. Raikh, and F. von Oppen, Phys. Rev. Lett. 96, 056803 (2006).

[14] A. S. Alexandrov, A. M. Bratkovsky, and R. S. Williams, Phys. Rev. B 67, 075301 (2003).

[15] P. S. Cornaglia, H. Ness, and D. R. Grempel, Phys. Rev. Lett. 93, 147201 (2004).

[16] L. Arrachea and M. J. Rozenberg, Phys. Rev. B 72, 041301 (2005).

[17] A. C. Hewson, The Kondo Problem to Heavy Fermions (Cambridge University Press, 1997).

[18] R. Bulla, T. Costi, and T. Pruschke, arXiv:condmat/0701105v1.

[19] A. C. Hewson and D. Meyer, J. Phys.:Condens. Matter 14, 427 (2002).

[20] K. G. Wilson, Rev. Mod. Phys. 47, 773 (1975).

[21] C. A. Balseiro, P. S. Cornaglia, and D. R. Grempel, Phys. Rev. B 74, 235409 (2006).

[22] T. Hotta, J. Phys. Soc. Jpn. 76, 084702 (2007).

[23] J. Paaske and K. Flensberg, Phys. Rev. Lett. 94, 176801 (2005).

[24] H. Pastawski, Phys. Rev. B 46, 4053 (1992).

[25] Y. Meir and N. S. Wingreen, Phys. Rev. Lett. 68, 2512 (1992).

[26] D. C. Langreth, Phys. Rev. 150, 516 (1966).

[27] P. S. Cornaglia, D. R. Grempel, and H. Ness, Phys. Rev. B 71, 075320 (2005).

[28] This expression is a consequence of Luttinger's theorem and has been shown to be exact for $g=0$ in the wideband limit [17, 26]. For $g \neq 0$ it was verified numerically and a computation of a few low-order diagrams in the expansion of the self-energy suggests that Luttinger's theorem and Eq. 6 can be generalized to the present situation [27]. 